\title{
An Iterative Algorithm to Build Chinese Language Models
}

\author{
Xiaoqiang Luo \\ Center for Language \\ and Speech Processing \\ The Johns Hopkins University \\ 3400 N. Charles St. \\ Baltimore, MD21218, USA \\ xiao@jhu.edu
}

\author{
Salim Roukos \\ IBM T. J. Watson Research Center \\ Yorktown Heights, NY 10598, USA \\ roukos@watson.ibm.com
}

\begin{abstract}
We present an iterative procedure to build a Chinese language model (LM). We segment Chinese text into words based on a word-based Chinese language model. However, the construction of a Chinese LM itself requires word boundaries. To get out of the chicken-and-egg problem, we propose an iterative procedure that alternates two operations: segmenting text into words and building an LM. Starting with an initial segmented corpus and an LM based upon it, we use a Viterbi-liek algorithm to segment another set of data. Then, we build an LM based on the second set and use the resulting LM to segment again the first corpus. The alternating procedure provides a self-organized way for the segmenter to detect automatically unseen words and correct segmentation errors. Our preliminary experiment shows that the alternating procedure not only improves the accuracy of our segmentation, but discovers unseen words surprisingly well. The resulting word-based LM has a perplexity of 188 for a general Chinese corpus.
\end{abstract}

\section{Introduction}

In statistical speech recognition(Bahl et al., 1983), it is necessary to build a language model $(\mathrm{LM})$ for assigning probabilities to hypothesized sentences. The LM is usually built by collecting statistics of words over a large set of text data. While doing so is straightforward for English, it is not trivial to collect statistics for Chinese words since word boundaries are not marked in written Chinese text. Chinese is a morphosyllabic language (DeFrancis, 1984) in that almost all Chinese characters represent a single syllable and most Chinese characters are also morphemes. Since a word can be multi-syllabic, it is generally non-trivial to segment a Chinese sentence into words(Wu and Tseng, 1993). Since segmentation is a fundamental problem in Chinese information processing, there is a large literature to deal with the problem. Recent work includes (Sproat et al., 1994) and (Wang et al., 1992). In this paper, we adopt a statistical approach to segment Chinese text based on an LM because of its autonomous nature and its capability to handle unseen words.

As far as speech recognition is concerned, what is needed is a model to assign a probability to a string of characters. One may argue that we could bypass the segmentation problem by building a characterbased LM. However, we have a strong belief that a word-based LM would be better than a characterbased ${ }^{1}$ one. In addition to speech recognition, the use of word based models would have value in information retrieval and other language processing applications.

If word boundaries are given, all established techniques can be exploited to construct an LM (Jelinek et al., 1992) just as is done for English. Therefore, segmentation is a key issue in building the Chinese LM. In this paper, we propose a segmentation algorithm based on an LM. Since building an LM itself needs word boundaries, this is a chicken-and-egg problem. To get out of this, we propose an iterative procedure that alternates between the segmentation of Chinese text and the construction of the LM. Our preliminary experiments show that the iterative procedure is able to improve the segmentation accuracy and more importantly, it can detect unseen words automatically.

In section 2, the Viterbi-like segmentation algorithm based on a LM is described. Then in section section:iter-proc we discuss the alternating procedure of segmentation and building Chinese LMs. We test the segmentation algorithm and the alternating procedure and the results are reported in sec-

\footnotetext{
${ }^{1}$ A character-based trigram model has a perplexity of 46 per character or $46^{2}$ per word (a Chinese word has an average length of 2 characters), while a word-based trigram model has a perplexity 188 on the same set of data. While the comparison would be fairer using a 5gram character model, that the word model would have a lower perplexity as long as the coverage is high.
} 
tion 4. Finally, the work is summarized in section 5 .

\section{2 segmentation based on LM}

In this section, we assume there is a word-based Chinese LM at our disposal so that we are able to compute the probability of a sentence (with word boundaries). We use a Viterbi-like segmentation algorithm based on the LM to segment texts.

Denote a sentence $S$ by $C_{1} C_{2} \cdots C_{n-1} C_{n}$, where each $C_{i}(1 \leq i \leq n\}$ is a Chinese character. To segment a sentence into words is to group these characters into words, i.e.

$$
\begin{aligned}
S= & C_{1} C_{2} \cdots C_{n-1} C_{n} \\
= & \left(C_{1} \cdots C_{x_{1}}\right)\left(C_{x_{1}+1} \cdots C_{x_{2}}\right) \\
& \cdots\left(C_{x_{m-1}+1} \cdots C_{x_{m}}\right) \\
= & w_{1} w_{2} \cdots w_{m}
\end{aligned}
$$

where $x_{k}$ is the index of the last character in $k^{\text {th }}$ word $w_{k}$, i,e $w_{k}=C_{x_{k-1}+1} \cdots C_{x_{k}}(k=1,2, \cdots, m)$, and of course, $x_{0}=0, x_{m}=n$.

Note that a segmentation of the sentence $S$ can be uniquely represented by an integer sequence $x_{1}, \cdots, x_{m}$, so we will denote a segmentation by its corresponding integer sequence thereafter. Let

$G(S)=\left\{\left(x_{1} \cdots x_{m}\right): 1 \leq x_{1} \leq \cdots \leq x_{m}, m \leq n\right\}$

be the set of all possible segmentations of sentence $S$. Suppose a word-based LM is given, then for a segmentation $g(S)=\left(x_{1} \cdots x_{m}\right) \in G(S)$, we can assign a score to $g(S)$ by

$$
\begin{aligned}
L(g(S)) & =\log P_{g}\left(w_{1} \cdots w_{m}\right) \\
& =\sum_{i=1}^{m} \log P_{g}\left(w_{i} \mid h_{i}\right)
\end{aligned}
$$

where $w_{j}=C_{x_{j-1}+1} \cdots C_{x_{j}}(j=1,2, \cdots, m)$, and $h_{i}$ is understood as the history words $w_{1} \cdots w_{i-1}$. In this paper the trigram model(Jelinek et al., 1992) is used and therefore $h_{i}=w_{i-2} w_{i-1}$

Among all possible segmentations, we pick the one $g^{*}$ with the highest score as our result. That is,

$$
\begin{aligned}
g^{*} & =\arg \max _{g \in G(S)} L(g(S)) \\
& =\arg \max _{g \in G(S)} \log P_{g}\left(w_{1} \cdots w_{m}\right)
\end{aligned}
$$

Note the score depends on segmentation $g$ and this is emphasized by the subscript in (9). The optimal segmentation $g^{*}$ can be obtained by dynamic programming. With a slight abuse of notation, let $L(k)$ be the max accumulated score for the first $k$ characters. $L(k)$ is defined for $k=1,2, \cdots, n$ with $L(1)=0$ and $L\left(g^{*}\right)=L(n)$. Given $\{L(i): 1 \leq i \leq k-1\}$, $L(k)$ can be computed recursively as follows:

$$
L(k)=\max _{1 \leq i \leq k-1}\left[L(i)+\log P\left(C_{i+1} \cdots C_{k} \mid h_{i}\right)\right]
$$

where $h_{i}$ is the history words ended with the $i^{\text {th }}$ character $C_{i}$. At the end of the recursion, we need to trace back to find the segmentation points. Therefore, it's necessary to record the segmentation points in $(10)$.

Let $p(k)$ be the index of the last character in the preceding word. Then

$$
p(k)=\arg \max _{1 \leq i \leq k-1}\left[L(i)+\log P\left(C_{i+1} \cdots C_{k} \mid h_{i}\right)\right]
$$

that is, $C_{p(k)+1} \cdots C_{k}$ comprises the last word of the optimal segmentation up to the $k^{t h}$ character.

A typical example of a six-character sentence is shown in table 1 . Since $p(6)=4$, we know the last word in the optimal segmentation is $C_{5} C_{6}$. Since $p(4)=3$, the second last word is $C_{4}$. So on and so forth. The optimal segmentation for this sentence is $\left(C_{1}\right)\left(C_{2} C_{3}\right)\left(C_{4}\right)\left(C_{5} C_{6}\right)$.

Table 1: A segmentation example

\begin{tabular}{c|cccccc}
\hline \hline chars & $C_{1}$ & $C_{2}$ & $C_{3}$ & $C_{4}$ & $\overline{C_{5}}$ & $C_{6}$ \\
\hline $\mathrm{k}$ & 1 & 2 & 3 & 4 & 5 & 6 \\
$\mathrm{p}(\mathrm{k})$ & 0 & 1 & 1 & 3 & 3 & 4 \\
\hline
\end{tabular}

The searches in (10) and (11) are in general timeconsuming. Since long words are very rare in Chinese $(94 \%$ words are with three or less characters (Wu and Tseng, 1993)), it won't hurt at all to limit the search space in (10) and (11) by putting an upper bound(say, 10) to the length of the exploring word, i.e, impose the constraint $i \geq \max 1, k-d$ in (10) and (11), where $d$ is the upper bound of Chinese word length. This will speed the dynamic programming significantly for long sentences.

It is worth of pointing out that the algorithm in (10) and (11) could pick an unseen word(i.e, a word not included in the vocabulary on which the LM is built on) in the optimal segmentation provided LM assigns proper probabilities to unseen words. This is the beauty of the algorithm that it is able to handle unseen words automatically.

\section{Iterative procedure to build LM}

In the previous section, we assumed there exists a Chinese word LM at our disposal. However, this is not true in reality. In this section, we discuss an iterative procedure that builds LM and automatically appends the unseen words to the current vocabulary.

The procedure first splits the data into two parts, set $T_{1}$ and $T_{2}$. We start from an initial segmentation of the set $T_{1}$. This can be done, for instance, by a simple greedy algorithm described in (Sproat et al., 1994). With the segmented $T_{1}$, we construct a $L M_{i}$ on it. Then we segment the set $T_{2}$ by using the $L M_{i}$ and the algorithm described in section 2 . At the same time, we keep a counter for each unseen word in optimal segmentations and increment the counter whenever its associated word appears in an 
optimal segmentation. This gives us a measure to tell whether an unseen word is an accidental character string or a real word not included in our vocabulary. The higher a counter is, the more likely it is a word. After segmenting the set $T_{2}$, we add to our vocabulary all unseen words with its counter greater than a threshold $c$. Then we use the augmented vocabulary and construct another $L M_{i+1}$ using the segmented $T_{2}$. The pattern is clear now: $L M_{i+1}$ is used to segment the set $T_{1}$ again and the vocabulary is further augmented.

To be more precise, the procedure can be written in pseudo code as follows.

Step 0: Initially segment the set $T_{1}$.

Construct an LM $L M_{0}$ with an initial vocabulary $V_{0}$

set $\mathrm{i}=1$.

Step 1: Let $\mathrm{j}=\mathrm{i} \bmod 2$;

For each sentence $S$ in the set $T_{j}$, do

1.1 segment it using $L M_{i-1}$.

1.2 for each unseen word in the optimal segmentation, increment its counter by the number of times it appears in the optimal segmentation.

Step 2: Let $A=$ the set of unseen words with counter greater than $c$.

set $V_{i}=V_{i-1} \cup A$.

Construct another $L M_{i}$ using the segmented set $T_{j}$ and the vocabulary $V_{i}$.

Step 3: $i=i+1$ and goto step 1 .

Unseen words, most of which are proper nouns, pose a serious problem to Chinese text segmentation. In (Sproat et al., 1994) a class based model was proposed to identify personal names. In (Wang et al., 1992), a title driven method was used to identify personal names. The iterative procedure proposed here provides a self-organized way to detect unseen words, including proper nouns. The advantage is that it needs little human intervention. The procedure provides a chance for us to correct segmenting errors.

\section{Experiments and Evaluation}

\subsection{Segmentation Accuracy}

Our first attempt is to see how accurate the segmentation algorithm proposed in section 2 is. To this end, we split the whole data set ${ }^{2}$ into two parts, half for building LMs and half reserved for testing. The trigram model used in this experiment is the standard deleted interpolation model described in (Jelinek et al., 1992) with a vocabulary of $20 \mathrm{~K}$ words.

Since we lack an objective criterion to measure the accuracy of a segmentation system, we ask three

\footnotetext{
${ }^{2}$ The corpus has about 5 million characters and is coarsely pre-segmented.
}

native speakers to segment manually 100 sentences picked randomly from the test set and compare them with segmentations by machine. The result is summed in table 2, where ORG stands for the original segmentation, P1, P2 and P3 for three human subjects, and TRI and UNI stand for the segmentations generated by trigram LM and unigram LM respectively. The number reported here is the arithmetic average of recall and precision, as was used in (Sproat et al., 1994), i.e., $1 / 2\left(\frac{n_{c}}{n_{1}}+\frac{n_{c}}{n_{2}}\right)$, where $n_{c}$ is the number of common words in both segmentations, $n_{1}$ and $n_{2}$ are the number of words in each of the segmentations.

Table 2: Segmentation Accuracy

\begin{tabular}{|c|c|c|c|c|c|c|}
\hline \hline & ORG & P1 & P2 & P3 & TRI & UNI \\
\hline ORG & & & & & 94.2 & 91.2 \\
\hline P1 & 85.9 & & & & 85.3 & 87.4 \\
\hline P2 & 79.1 & 90.9 & & & 80.1 & 82.2 \\
\hline P3 & 87.4 & 85.7 & 82.2 & & 85.6 & 85.7 \\
\hline
\end{tabular}

We can make a few remarks about the result in table 2. First of all, it is interesting to note that the agreement of segmentations among human subjects is roughly at the same level of that between human subjects and machine. This confirms what reported in (Sproat et al., 1994). The major disagreement for human subjects comes from compound words, phrases and suffices. Since we don't give any specific instructions to human subjects, one of them tends to group consistently phrases as words because he was implicitly using semantics as his segmentation criterion. For example, he segments the sentence ${ }^{3}$ dao4 jia1 1 i2 chi1 dun4 fan 4 (see table 3) as two words dao4 jia1 1 i2 (go home) and chi1 dun4 fan4(have a meal) because the two "words" are clearly two semantic units. The other two subjects and machine segment it as dao4 / jia1 li2/ chil/ dun4 / fan4.

Chinese has very limited morphology (Spencer, 1991) in that most grammatical concepts are conveyed by separate words and not by morphological processes. The limited morphology includes some ending morphemes to represent tenses of verbs, and this is another source of disagreement. For example, for the partial sentence zuo4 wan2 le, where le functions as labeling the verb zuo4 wan2 as "perfect" tense, some subjects tend to segment it as two words zuo4 $\operatorname{wan} 2 /$ le while the other treat it as one single word.

Second, the agreement of each of the subjects with either the original, trigram, or unigram segmentation is quite high (see columns 2, 6, and 7 in Table 2) and appears to be specific to the subject.

\footnotetext{
${ }^{3}$ Here we use Pin Yin followed by its tone to represent a character.
} 
Third, it seems puzzling that the trigram LM agrees with the original segmentation better than a unigram model, but gives a worse result when compared with manual segmentations. However, since the LMs are trained using the presegmented data, the trigram model tends to keep the original segmentation because it takes the preceding two words into account while the unigram model is less restricted to deviate from the original segmentation. In other words, if trained with "cleanly" segmented data, a trigram model is more likely to produce a better segmentation since it tends to preserve the nature of training data.

\subsection{Experiment of the iterative procedure}

In addition to the 5 million characters of segmented text, we had unsegmented data from various sources reaching about 13 million characters. We applied our iterative algorithm to that corpus.

Table 4 shows the figure of merit of the resulting segmentation of the 100 sentence test set described earlier. After one iteration, the agreement with the original segmentation decreased by 3 percentage points, while the agreement with the human segmentation increased by less than one percentage point. We ran our computation intensive procedure for one iteration only. The results indicate that the impact on segmentation accuracy would be small. However, the new unsegmented corpus is a good source of automatically discovered words. A 20 examples picked randomly from about 1500 unseen words are shown in Table 5. 16 of them are reasonably good words and are listed with their translated meanings. The problematic words are marked with "?".

\subsection{Perplexity of the language model}

After each segmentation, an interpolated trigram model is built, and an independent test set with 2.5 million characters is segmented and then used to measure the quality of the model. We got a perplexity 188 for a vocabulary of $80 \mathrm{~K}$ words, and the alternating procedure has little impact on the perplexity. This can be explained by the fact that the change of segmentation is very little (which is reflected in table reftab:accuracy-iter ) and the addition of unseen words $(1.5 \mathrm{~K})$ to the vocabulary is also too little to affect the overall perplexity. The merit of the alternating procedure is probably its ability to detect unseen words.

\section{Conclusion}

In this paper, we present an iterative procedure to build Chinese language model(LM). We segment Chinese text into words based on a word-based Chinese language model. However, the construction of a Chinese LM itself requires word boundaries. To get out of the chicken-egg problem, we propose an iterative procedure that alternates two operations: segmenting text into words and building an LM. Starting with an initial segmented corpus and an LM based upon it, we use Viterbi-like algorithm to segment another set of data. Then we build an LM based on the second set and use the LM to segment again the first corpus. The alternating procedure provides a self-organized way for the segmenter to detect automatically unseen words and correct segmentation errors. Our preliminary experiment shows that the alternating procedure not only improves the accuracy of our segmentation, but discovers unseen words surprisingly well. We get a perplexity 188 for a general Chinese corpus with 2.5 million characters ${ }^{4}$.

\section{Acknowledgment}

The first author would like to thank various members of the Human Language technologies Department at the IBM T.J Watson center for their encouragement and helpful advice. Special thanks go to Dr. Martin Franz for providing continuous help in using the IBM language model tools. The authors would also thank the comments and insight of two anonymous reviewers which help improve the final draft.

\section{References}

Richard Sproat, Chilin Shih, William Gale and Nancy Chang. 1994. A stochastic finite-state word segmentation algorithm for Chinese. In Proceedings of $A C L ' 94$, pages 66-73

Zimin Wu and Gwyneth Tseng 1993. Chinese Text Segmentation for Text Retrieval: Achievements and Problems Journal of the American Society for Information Science, 44(9):532-542.

John DeFrancis. 1984. The Chinese Language. University of Hawaii Press, Honolulu.

Frederick Jelinek, Robert L. Mercer and Salim Roukos. 1992. Principles of Lexical Language Modeling for Speech recognition. In Advances in Speech Signal Processing, pages 651-699, edited by S. Furui and M. M. Sondhi. Marcel Dekker Inc., 1992

L.R Bahl, Fred Jelinek and R.L. Mercer. 1983. A Maximum Likelihood Approach to Continuous Speech Recognition. In IEEE Transactions on Pattern Analysis and Machine Intelligence, 1983,5(2):179-190

Liang-Jyh Wang, Wei-Chuan Li, and Chao-Huang Chang. 1992. Recognizing unregistered names for mandarin word identification. In Proceedings of COLING-92, pages 1239-1243. COLING

\footnotetext{
${ }^{4}$ Unfortunately, we could not find a report of Chinese perplexity for comparison in the published literature concerning Mandarin speech recognition
} 
Andrew Spencer. 1992. Morphological theory : an introduction to word structure in generative grammar pages 38-39. Oxford, UK ; Cambridge, Mass., USA. Basil Blackwell, 1991.
Table 3: Segmentation of phrases

\begin{tabular}{c|ccccc}
\hline Chinese & dao4 & jial li2 & chil & dun4 & fan4 \\
Meaning & go & home & eat & a & meal \\
\hline
\end{tabular}

Table 4: Segmentation of accuracy after one iteration

\begin{tabular}{|c|cc|}
\hline \hline & TR0 & TR1 \\
ORG & .920 & .890 \\
P1 & .863 & .877 \\
P2 & .817 & .832 \\
P3 & .850 & .849 \\
\hline
\end{tabular}

Table 5: Examples of unseen words

\begin{tabular}{|c|c|}
\hline PinYin & Meaning \\
\hline $\begin{array}{r}\text { kui2 er2 } \\
\text { he2 shi4 lu4 yin1 dai4 } \\
\text { shou2 dao3 } \\
\text { ren4 zhong4 } \\
\text { ji4 jian3 } \\
\text { zi4 hai4 } \\
\text { shuang1 bao3 } \\
\text { ji4 dong1 } \\
\text { zi3 jiao1 } \\
\text { xiao1 long2 shi2 } \\
\text { li4 bo4 hai3 } \\
\text { du4 shan1 } \\
\text { shang1 ban4 } \\
\text { liu6 hai4 } \\
\text { sa4 he4 le4 } \\
\text { kuai4 xun4 } \\
\text { cheng4 jing3 } \\
\text { huang2 du2 } \\
\text { ba3 lian2 } \\
\text { he2 dao3 }\end{array}$ & $\begin{array}{l}\text { last name of former US vice president } \\
\text { cassette of audio tape } \\
\text { (abbr)pretect (the) island } \\
\text { first name or part of a phrase } \\
\text { (abbr) discipline monitoring } \\
\text { ? } \\
\text { double guarantee } \\
\text { (abbr) Eastern He Bei province } \\
\text { purple glue } \\
\text { personal name } \\
\text { ? } \\
\text { ? } \\
\text { (abbr) commercial oriented } \\
\text { six (types of) harms } \\
\text { translated name } \\
\text { fast news } \\
\text { train cop } \\
\text { yellow poison } \\
\text { ? } \\
\text { a (biological) jargon }\end{array}$ \\
\hline
\end{tabular}

